\title{
Improvement of transient stability by NAS battery system installation on a generator bus
}

\author{
Yasunori Tomita, Masaya Takiguchi, Shinichi Iwamoto \\ Waseda University,3-4-1 Ohkubo Shinjyu-ku, Tokyo 169-8555, Japan
}

\begin{abstract}
Recently, because of the introduction of renewable energies, the electric power system has become larger and more complex. Under such environment, the number of transmission lines has increased, on which heavy-load currents flow, and as the result, the transient stability has emerged as a significant problem. In this paper, we propose a smart approach in which we use the NAS battery system on a generator bus for improving transient stability, and determine the output of NAS battery system by the LQR control theory. Finally, we verify the effects of the proposed method for the transient stability on the IEEJ EAST 10-machine 47-bus system model.
\end{abstract}

Keywords: Transient stability, NAS battery system, linear quadratic regulator, generator bus

\section{Introduction}

Recently, because of the introduction of renewable energies such as wind power generations and PV generations, electric power systems have become larger and more complex. Under such environment, it is more difficult to keep transient stability in the system without generator stepping-out when a severe fault occurs on a certain transmission line. Therefore, transient stability analysis becomes more important.

The use of battery systems such as NAS battery systems have been discussed and made realizable for the electric power systems [1]-[4]. Until now, the main purpose of their use has been load fluctuation leveling and stabilization of the renewable energies outputs. However, it will be possible to use them also for the improvement of transient stability of Generator if the capacity gets larger and the output becomes more high-speed.

Therefore, in this paper, we assume that a wind power generation with a battery system which can be controlled at high-speed (NAS battery system, in this paper) is installed on a generator bus in the future, and propose to use the NAS battery system also for improving the transient stability of Generator when a severe fault occurs. Concretely, we simulate a three-phase to ground fault of one line in double circuit transmission lines. Then, a NAS battery system on a critical generator bus will activate to charge the electrical output of the generator to avoid its stepping-out. Moreover, we decide the NAS battery system output with linear quadratic regulator (LQR) control theory [5] and state-feedback regulator accounting for delay [6]. Then, we take into account the generator states (rotor angle, generator speed, internal voltage, field voltage). We use only the local critical generator state, and perform a local control [7].

Finally, we run simulations on the IEEJ EAST 10-machine 47-bus system model [8] to verify the effect of the proposed method for the transient stability improvement of Generator.

\section{Basic Theory}

In this section, the concept of LQR control theory [5] and state-feedback regulator accounting for delay [6] are briefly described.

\footnotetext{
* Manuscript received May 15, 2013; revised July 29, 2013.

Corresponding author Tel.: +81 3-5286-3184; E-mail address: takiguchi.masaya.pwrs@gmail.com.
} 


\subsection{Linear quadratic regulator (LQR) control theory [5]}

A state equation can be written as Eq. (1).

$$
\dot{x}=A x+B u
$$

$x$ : state values, $u$ : control input

In this theory, the control input minimizes the following evaluation function.

$$
J=\frac{1}{2} \int_{0}^{\infty}\left(x^{T} Q x+u^{T} R u\right) d t
$$

where $Q$ and $R$ are positive diagonal weighting matrices.

To obtain the best amount $u^{*}$ for the control, we considered the constraints. The present system is given by Eq.(3) and the best control for this system is written as in Eq. (4).

$$
\begin{aligned}
& A x+B u=F \\
& A x+B u^{*}=\dot{x}
\end{aligned}
$$

To bring the control of Eq. (3) close to the control of Eq. (4), the following condition is added.

$$
F-\dot{x}=g
$$

When the Lagrange multiplier is expressed as $Z$, the evaluation function of Eq. (2) becomes as follows.

$$
J=\frac{1}{2} \int\left(x^{T} Q x+u^{T} R u\right) d t+\int Z^{T} g d t
$$

The amount $u$ of the operation must be used to bring $g$ close to 0 in order to minimize this evaluation function.

When the transformation is carried out, the amount of the best control is given by Eq. (7).

$$
u^{*}=-R^{-1} B^{T} P x=-K x
$$

where $\mathrm{P}$ is obtained from the Ricatti equation (8).

$$
P A-P B R^{-1} B^{T} P+Q+A^{T} P=0
$$

\subsection{A State-feedback Regulator accounting for Delay [6]}

In the preceding section, we described LQR Control Theory in a continuous-time linear system. However, in proposed method, we determine the NAS battery system output every $10 \mathrm{~ms}$, therefore we have to describe the theory with a discrete-time linear system.

In the discrete-time linear system, the entire continuous-time interval is set with the sample time $T$. In the sample time $T$, the phenomena are regarded as the zero-order hold (phenomena constancy). If the sample time $T$ is very short, the error can be disregarded. The discrete-time linear system equation and the best control are shown in Eq.(9), (10), respectively.

$$
\begin{aligned}
& x[i+1]=A^{\prime} x[i]+B^{\prime} u[i] \\
& u[i]=-K^{\prime} x[i]
\end{aligned}
$$

The best control in Eq. (10) can be calculated with an assumption that we can get all state values in real-time. However, in stabilizing control in an electric power system, delay exists because of measurements and data transmissions. These delays cannot be disregarded. Thus, a time delay is needed in the design of the state-feedback regulator.

Therefore, the state-feedback regulator is given by (11) in order to account for the $L$ sample delay in the discrete-time linear system equation with a theoretical guarantee. 


$$
\begin{gathered}
u[i]=-K^{\prime} A^{\prime L} x[i-L]-K^{\prime} A^{\prime L-1} B^{\prime} u[i-L]-K^{\prime} A^{\prime L-2} B^{\prime} u[i-1]-\cdots \\
\cdots-K^{\prime} A^{\prime} B^{\prime} u[i-2]-K^{\prime} B^{\prime} u[i-1]
\end{gathered}
$$

From Eq. (11), we can see that the best control $u[i]$ is calculated with known values $x[i-L]$ and $u[i-1] \ldots u[i-L]$.

\section{Proposed Method}

In this section, we propose to improve transient stability of generator by using a NAS battery system installed on a generator bus.

\subsection{Outline of proposed method}

The generator equations of generator $i$ concerning the rotor angle and the generator speed are given in Eq. (12) and Eq. (13), respectively.

$$
\begin{aligned}
& \dot{\delta}_{(i)}=\omega_{0}\left(\omega_{(i)}-1\right) \\
& M_{(i)} \dot{\delta}_{(i)}=P_{m(i)}-P_{e(i)}-D_{(i)}\left(\omega_{(i)}-1\right)
\end{aligned}
$$

When a severe fault occurs on a certain transmission line, the fault is cleared by the circuit breaker (CB), and an electrical output $P_{e}$ of the generator $i$ which is located near the fault point goes down suddenly. Then, from Eq. (12) and Eq. (13), the balance of the mechanical input $P_{m}$ and the electrical output $P_{e}$ of the generator $i$ is broken, and the generator speed accelerates. As the result, the rotor angle excesses a certain threshold, and we judge the generator $i$ as stepped-out.

To suppress the generator speed acceleration and improve the transient stability of Generator, we propose to use a NAS battery system installed on the critical generator bus, and charge the battery with the electrical output $P_{e}$ immediately. We add the NAS battery system output $P_{b s}$ to the electrical output $P_{e}$ to have $P_{e, \text { new(i) }}$ as in Eq.(14) and (15).

$$
\begin{aligned}
M_{(i)} \dot{\omega}_{(i)} & =P_{m(i)}-P_{e, \text { new }(i)}-D_{(i)}\left(\omega_{(i)}-1\right) \\
P_{e, \text { new }(i)} & =P_{e(i)}-P_{b s}
\end{aligned}
$$

where $P_{b s}$ is the NAS battery system output [pu] (negative when the NAS battery system is charging.)

When we use battery systems such as NAS battery systems, we can suppress an imbalance between a mechanical input and an electrical output of a generator drastically, and can control a rotor angle acceleration and deceleration. Therefore, the proposed method seems to be effective means for improving transient stability of Generator. The application of battery systems on generator buses seems to be useful also for the problem of voltage and frequency deviations when the electric powers generated by renewable energies are increased. Concept of the proposed method is shown in Fig. 1.

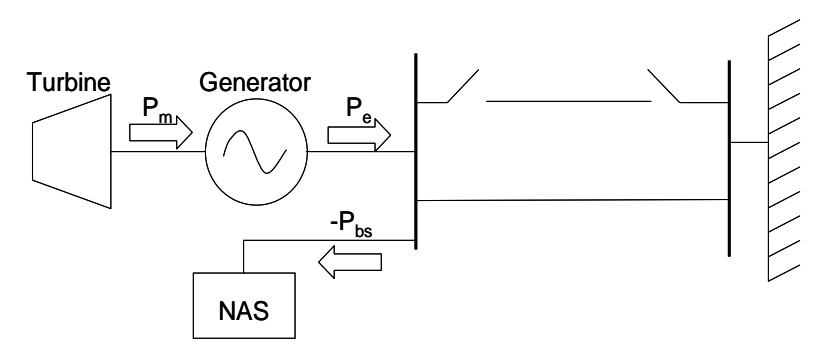

Fig. 1. Concept of the proposed method

\subsection{Decision on the Output of the NAS Battery system [5],[8]}

In this paper, we apply LQR Control Theory so as to determine the control input, i.e. the output of the 
NAS battery system. With this theory, we can obtain the optimal output which not only controls the acceleration and deceleration of the rotor angle but also recovers the generator state to the first condition sooner.

At first, we make a state equation Eq. (1). In this paper, when deciding the NAS battery system output, we consider a generator state (rotor angle, generator speed, internal voltage, field voltage) of the generator $i$, and write the state value vector $X$ with deviations of each generator state as Eq. (16).

$$
x=\left[\Delta \delta_{(i)}, \Delta \omega_{(i)}, \Delta E_{(q i)}^{\prime}, \Delta E_{f d(i)}\right]^{T}
$$

where $\Delta \delta_{(i)}$ is the deviation of rotor angle [rad], $\Delta \omega_{(i)}$ is the deviation of generator speed [pu], $\Delta E_{q(i)}^{\prime}$ is the deviation of internal voltage [pu], and $\Delta E_{f d(i)}$ is the deviation of field voltage [pu].

In addition, the control input $u$ represents the NAS battery system output.

$$
u=P_{b s}
$$

Then, firstly, we decide the matrix A. By approximating generator equations (equations of rotor angle, generator speed, internal voltage, and field voltage), we obtain Eq. (18) to (21).

$$
\begin{aligned}
& \Delta \dot{\delta}_{(i)}=\omega_{0} \Delta \omega_{(i)} \\
& \Delta \dot{\omega}=-\frac{K_{1}}{M_{(i)}} \Delta \delta_{(i)}-\frac{D_{(i)}}{M_{(i)}} \Delta \omega_{(i)}-\frac{K_{2}}{M_{(i)}} \Delta E_{q(i)}^{\prime} \\
& \Delta \dot{E}_{q(i)}^{\prime}=-\frac{K_{3}}{\tau_{d 0(i)}^{\prime}} \Delta \delta-\frac{K_{4}}{\tau_{d 0(i)}^{\prime}}+\frac{1}{\tau_{d 0(i)}^{\prime}} \Delta E_{f d(i)} \\
& \Delta \dot{E}_{f d(i)}=-\frac{K_{a(i)} K_{5}}{\tau_{a(i)}} \Delta \delta(i)-\frac{K_{a(i)} K_{6}}{\tau_{a(i)}} \Delta E_{q(i)}^{\prime}-\frac{1}{\tau_{a(i)}} \Delta E_{f d(i)}
\end{aligned}
$$

where $K_{a(i)}, \tau_{d 0(i)}^{\prime}, \tau_{a(i)}$ are a AVR gain, rotor time constant, and AVR time constant, respectively. $K_{1} \sim K_{6}$ are constant values related to the electric power system, which can be calculated from the characteristics of generator $i$, operation conditions and system state. From Eq. (18) to (21), we obtain the matrix $A$ as in Eq. (22).

$$
A=\left[\begin{array}{cccc}
0 & \omega_{0} & 0 & 0 \\
-\frac{K_{1}}{M_{(i)}} & -\frac{D_{(i)}}{M_{(i)}} & -\frac{K_{2}}{M_{(i)}} & 0 \\
-\frac{K_{3}}{\tau_{d 0(i)}^{\prime}} & 0 & -\frac{1}{\tau_{d 0(i)}^{\prime} K_{4}} & \frac{1}{\tau_{d 0(i)}^{\prime}} \\
-\frac{K_{a(i)} K_{5}}{\tau_{a(i)}} & 0 & -\frac{K_{a(i)} K_{6}}{\tau_{a(i)}} & -\frac{1}{\tau_{a(i)}^{\prime}}
\end{array}\right]
$$

Secondly, we make the matrix $B$. We assume that the output of the NAS battery system has an influence on only a generator speed, and set the matrix $B$ as Eq.(23).

$$
B=\left[0,1 / M_{(i)}, 0,0\right]^{T}
$$

With Eqs.(16), (17), (22) and (23), we obtain the best control Eq.(11) which considers the generator state as the output of the NAS battery system.

\section{Simulations}

In this section, we run simulations for three-phase to ground faults using the IEEJ EAST 10- machine 47-bus system model (Fig.2), to verify the validity of the proposed method. 


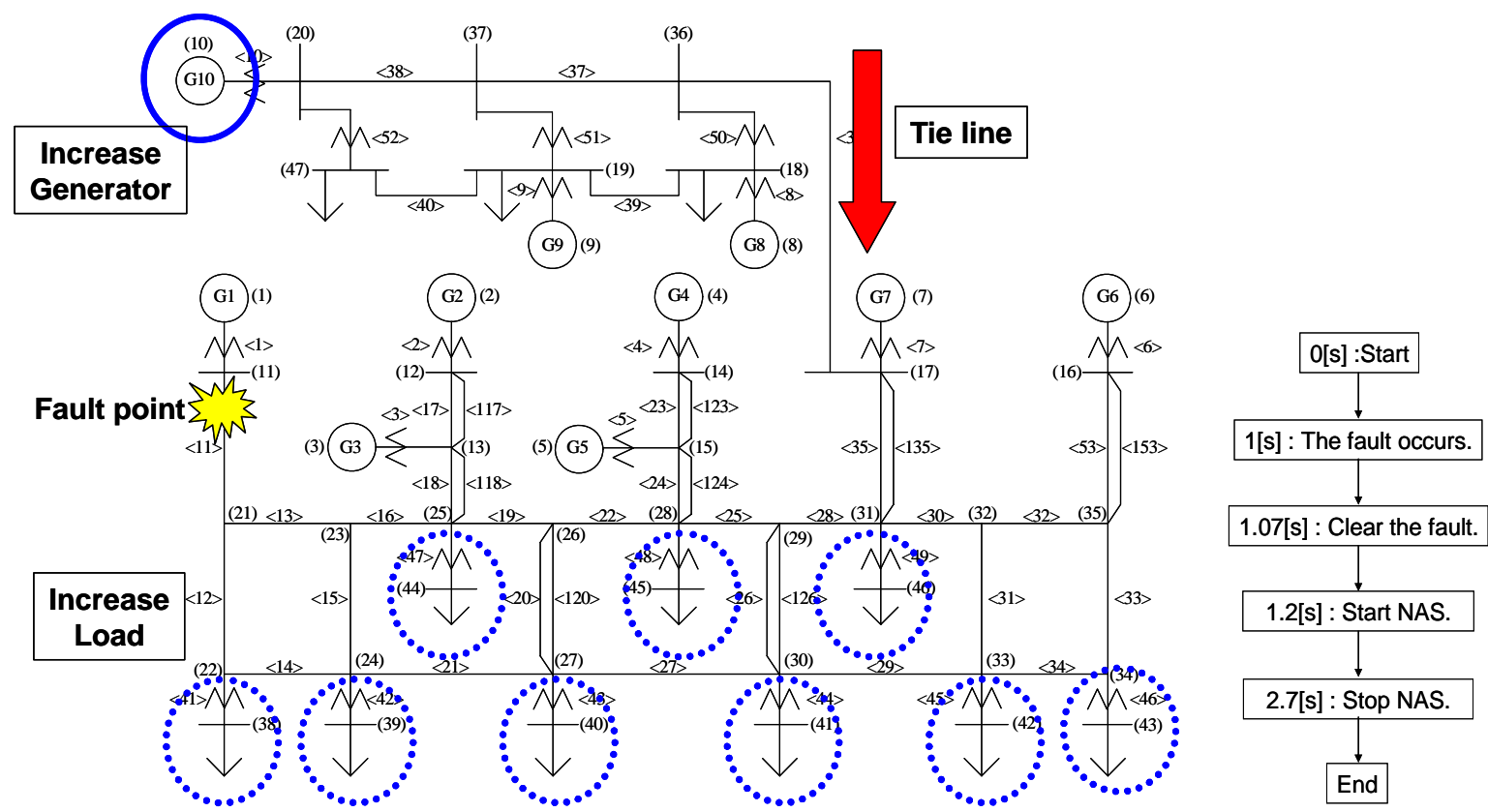

Fig. 2. IEEJ EAST 10-machine 47-bus system model [8].

Fig. 3. Flowchart.

In the simulations, the base value is 1000 MVA, and the load ratio is 0.80 . The severest fault on the double circuit transmission line 11-21 near bus 11 is considered. As for the fault case, one circuit of the double circuit line is opened $0.07 \mathrm{~s}\left(=t_{\mathrm{cl}}\right)$ after the fault. We calculate the rotor angles every $10 \mathrm{~ms}$ by Runge-Kutta method, and regard the generator as stepped-out when a rotor angle exceeds $180^{\circ}$.

In addition, in this paper, we set the output of the NAS battery system as $0.1 \mathrm{pu}$. The NAS battery system starts to output at $200 \mathrm{~ms}$ after the fault, and continue to output for $1.5 \mathrm{~s}$. The delays of measurements and data transmissions are $40 \mathrm{~ms}$ (4 samples) in all, and use the state-feedback regulator to determine the best control Eq. (11). The flowchart is shown in Fig. 3. Conditions of simulations are shown in Table 1.

Table 1. Conditions

\begin{tabular}{cccc}
\hline \hline Section & Base & Load ratio & $t_{\mathrm{cl}}$ \\
\hline day & $1000 \mathrm{MVA}$ & 0.80 & $0.07 \mathrm{~s}$ \\
\hline \hline
\end{tabular}

\subsection{The severest fault on transmission line 11-21 near bus 11}

In these simulations, we consider the severest fault on the transmission line 11-21 near bus 11 . The fault has a great influence on Generator 1 which is located near the fault point. In this situation, Generator 1 is inclined to stepped-out sooner than the other generators.

Then, we consider using a NAS battery system located on Generator Bus 11 in order to prevent stepped-out of Generator 1, and to improve transient stability of Generator 1. In addition, we use LQR control theory to determine the NAS battery system output. In case of this, actually, we regard it as impossible to determine the NAS battery system output every $10 \mathrm{~ms}$ using all generator states [7]. Therefore, we use only the generator state of Generator 1, and perform a local control.

\subsection{Results of simulations}

1) The NAS battery system output

The change of the NAS battery system output when the tie-line flow is 3.6 pu is shown in Fig. 4. From Fig. 4, we can see that the NAS battery system starts charging the electric power after $200 \mathrm{~ms}$ from the fault occurrence, and that it continues charging for $1.5 \mathrm{~s}$ to control the rotor angle acceleration of Generator 1. 


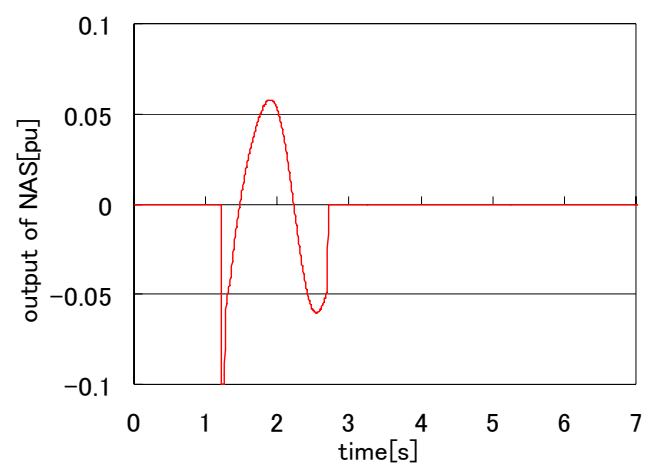

Fig. 4. The NAS battery system output

2) The state of Generator 1

In this section, we compare the state (rotor angle, generator speed, internal voltage, field voltage) of Generator 1 when we do not set the NAS battery system, with the state when we set the NAS battery system with LQR control theory (the proposed method). We show the figure of both generator states when the tie-line flow is 3.71 pu in Figs. 5 to 8.

From Figs. 5 to 8, by using the proposed method, we can see that the first transient of all generator state variables are restrained and the convergences to their first conditions become sooner. These results are obtained by the effects of the proposed method. By deciding the NAS battery system output by LQR control theory, the deviations of the generator state are controlled.

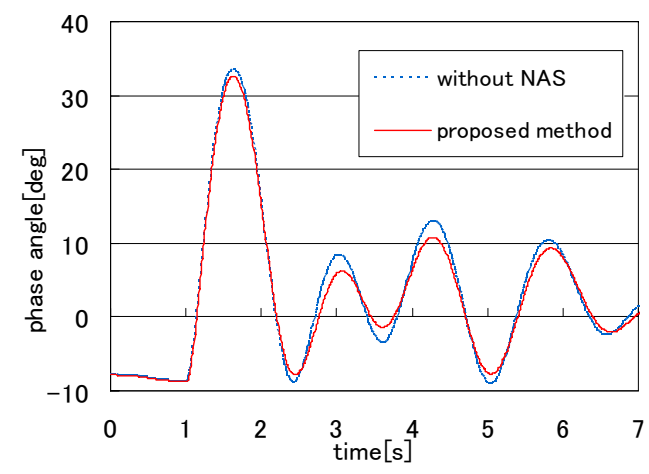

Fig. 5. Rotor Angle $\delta_{(1)}$

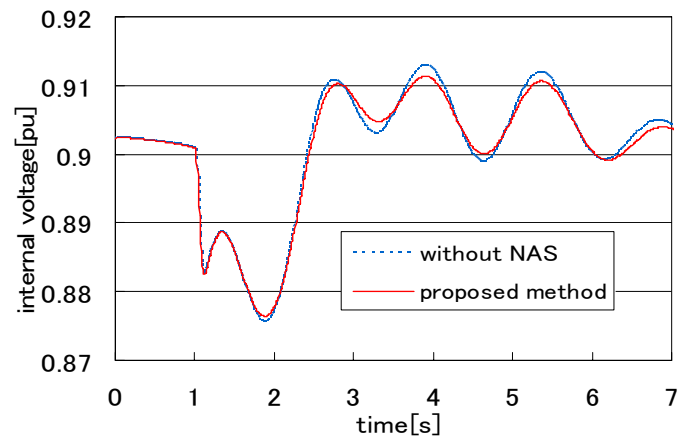

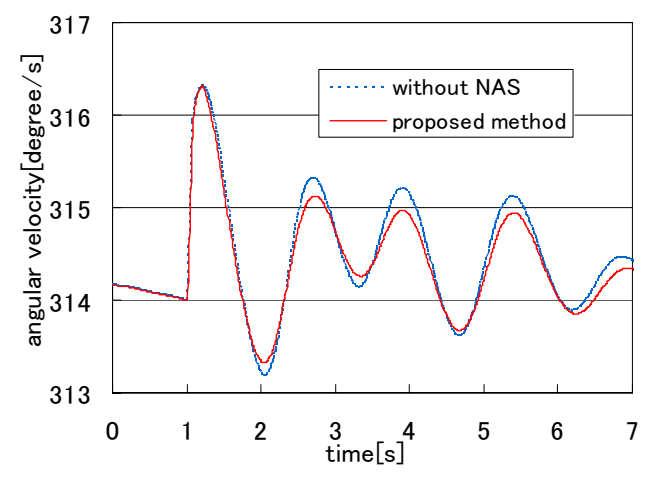

Fig. 6. Generator Speed $\omega_{(1)}$

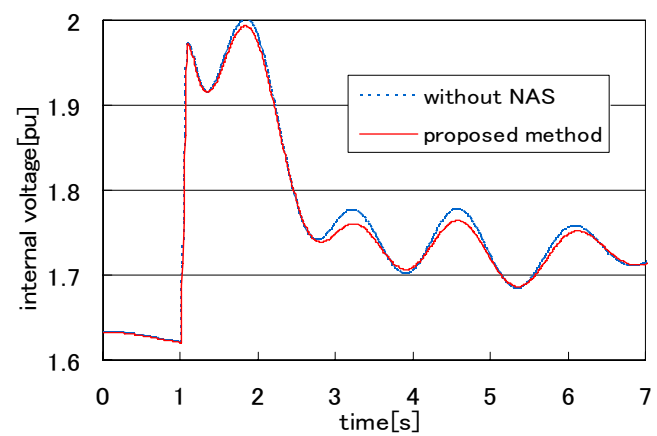

Fig. 7. Internal Voltage $E_{\text {\&1) }}^{\prime}$

Fig. 8. Field Voltage $E_{f d(1)}$

\section{Conclusion}

In this paper, we assume that a wind power generation with a NAS battery system is installed on a generator bus as a future scenario, and propose a smart approach in which we use a NAS battery system 
for the improvement of transient stability of Generator. By using the NAS battery system, we expect that we can suppress an imbalance between mechanical input and electrical output of a generator drastically, and can improve the transient stability of Generator. Additionally, we control the NAS battery system output by LQR Control Theory to consider the generator state. We use only the local critical generator state to perform a local control.

Finally, we run simulations on the IEEJ EAST 10-machine 47-bus system model. By using the proposed method, the deviations of generator state values in Generator 1 are controlled. Therefore, we confirm the validities of the proposed method for improving transient stability of Generator, and, moreover, for achieving the economical advantage of tie-line and the increase of renewable energy introduction.

\section{References}

[1] Hirabayashi S, Tomita Y, Iwamoto S. Enhancement of transient stability ATC using NAS battery systems. In: Proc. of IEEE/PES Transmission and Distribution Conference and Exposition, 2008:1-6.

[2] Ohtaka T, Iwamoto S. A Study of Dynamic Voltage Control Using NAS Batteries. Presented at: IEEJ National Convention, 2001.

[3] Demirci O, Nichols D, Tamyurek B. The NAS battery: A multi-function energy storage system. Presented at: IEEE/PES 2003 General Meeting Conference Proceedings, 2003.

[4] Tomita Y, Iwamoto S. Improving of transient stability ATC by rescheduling of generations and use of battery systems. National Convention IEEJ

[5] Nishida K, Hirabayashi S, Iwamoto S. Operation strategy of TCSC considering stability ATC. In Proc. of 2006 IEEE PES Power Systems Conference and Exposition, 2006: 705-710.

[6] Mita T. Digital Control Theory. Shokodo, 1991

[7] Sata T. GPS Measurement Technology. Ohmsha, 2003

[8] Kunitomo O, Iwamoto S. Power system stabilizing control with unified power flow controller using global positioning system. In: Proc. of IEEJ Power Engineering and Power System Engineering Symposium, 1999:55-60. 